\title{
Sigmoid carcinoma: A rare presentation and diagnostic challenge
}

\author{
Bassem Amr, Komal Munir, Natasha Santana-Vaz, \\ Venkateswarlu Velineni
}

\begin{abstract}
Introduction: Adenocarcinoma represents less than $2 \%$ of all bladder carcinomas. Metastatic adenocarcinoma is the most common form and usually represents a direct extension from a primary lesion either in the colon, prostate or the female genital organs. Other categories include primary vesical or urachal. Case Report: We present a case of 79-yearold female presented with microscopic heamaturia, frequency, urgency and weight loss. An adenocarcinoma of the urinary bladder of intestinal origin was founded. Conclusion: Primary adenocarcinoma of the urinary bladder is uncommon neoplasm and the reported incidence of the primary adenocarcinoma is 0.5$2 \%$. It represents a diagnostic challenge raising the inquiry about the site of the lesion. Three major classes were identified: primary vesical adenocarcinoma, urachal adenocarcinoma and extravesical adenocarcinoma involving the bladder. The metastatic adenocarcinoma is the most common category representing a direct extension from a nearby organ or metastatic spread. The most common treatment for urinary bladder adenocarcinoma is surgery. Generally speaking, treatment options include: surgery, radiotherapy, chemotherapy and immunotherapy. Metastatic adenocarcinoma
\end{abstract}

Bassem Amr ${ }^{1}$, Komal Munir ${ }^{1}$, Natasha Santana-Vaz ${ }^{1}$, Venkateswarlu Velineni ${ }^{1}$

Affiliations: 'Worcestershire Acute Hospitals NHS Trust, Woodrow Drive, Redditch, B98 7UB.

Corresponding Author: Mr. Bassem Amr, Worcestershire Acute Hospitals NHS Trust, Woodrow Drive, Redditch, B98 7UB. Ph: +44 152750 3030, Mob: +44 78256356 24; Email: dr_bassem277@yahoo.com

Received: 23 July 2012

Accepted: 23 February 2013

Published: 01 September 2013 to the urinary bladder especially of colonic origin is of particular interest and need to be ruled out before making a diagnosis of primary adenocarcinoma of urinary bladder.

Keywords: Bowel cancer, Adenocarcinoma, Bladder tumor

$* * * * * * * * *$

Amr B, Munir K, Santana-Vaz N, Velineni V. Sigmoid carcinoma: A rare presentation and diagnostic challenge. International Journal of Case Reports and Images 2013;4(9):477-480.

$* * * * * * * * *$

doi:10.5348/ijcri-2013-09-36o-CR-4

\section{INTRODUCTION}

Adenocarcinoma represents less than 2\% of all bladder carcinomas [1]. Primary adenocarcinoma is an uncommon malignant neoplasm and is a source of a diagnostic challenge. It is less frequently encountered in areas where bilharziasis is endemic whereas the squamous cell carcinoma is more prevalent. This incidence ranges between $5-11.4 \%$. Chronic vesical irritation and infection are predisposing factors for metaplastic changes of the urothelium [2]. Based on the original tumor site, adenocarcinoma of the bladder could be classified into three categories: primary, urachal and metastatic. Primary vesical adenocarcinoma needs to be distinguished from the more common extravesical metastatic adenocarcinoma (direct spread, lymphatic, and hematogenous). The principal primary organs to be considered include prostate, colon, female genital tract, appendix, stomach and breast. Clinical association with bladder exstrophy and schistosomiasis has been well documented. Clinical assessment, imaging, histologic, and immunohistochemical correlation should be done 
while investigating this diagnosis due to histologic and immunohistochemical overlapping between primary bladder adenocarcinoma and metastatic adenocarcinoma [3]. Prognosis and therapeutic options for primary versus metastatic adenocarcinoma vary widely.

\section{CASE REPORT}

A 79-year-old female was presented to her general practitioner in January 2012 with urinary symptoms in the form of frequency, urgency and passing dark urine. She had also noted recent weight loss of $3 \mathrm{~kg}$. Urine dipstick was positive for leukocytes, protein and blood. Antibiotic was prescribed by her doctor for urinary tract infection. The symptoms recurred once the course of treatment was completed; for which she was given three separate courses of antibiotics as her mid-stream urine samples showed E. coli. Her ultrasound scan of the urinary tract showed a simple cyst on the right kidney and incidental finding of a bulky uterus; therefore pelvic scan was undertaken. A retroverted bulky fibroid uterus measuring $6.5 \times 2.9 \times 4.6 \mathrm{~cm}$ was revealed. A heterogenous myometrium demonstrating echogenic foci throughout anteriorly was founded and a well-defined echogenic area within the left adnexa measuring $2.5 \mathrm{~cm}$ with small amount of free fluid was seen on the scan. She was referred to the hematuria clinic on April 2012, where a flexible cystoscopy was performed under local anesthesia. There was an inverted granuloma present at the trigone and an area of inflammation on the right-hand side of the posterior wall. Computed tomography (CT) scan of the abdomen and pelvis showed marked sigmoid colon thickening with associated diverticulosis suggestive of complicated sigmoid diverticulitis with a localized perforation. Also a focal urinary bladder wall thickening was likely secondary to the colonic abnormality with no definitive evidence of a colovesical fistula on this scan (Figure 1). She was admitted to our hospital for cystoscopy and biopsy. Examination under anesthesia revealed a $5-\mathrm{cm}$ pelvic mass extending into the left iliac fossa. Cystoscopy revealed a lesion in the trigone (Figure 2) which was biopsied. Histopathological findings of the cystoscopic biopsy revealed moderately differentiated adenocarcinoma of an intestinal appearance infiltrating bladder mucosa. Her case was discussed at the urology and colorectal multidisciplinary team (MDT) meetings. A staging CT scan showed a sigmoid colon tumor, possibly involving the roof of the bladder with lymphadenopathy and no evidence of distant metastatic disease. Her blood investigations were within normal limits before she was scheduled for anterior resection, partial cystectomy and total abdominal hysterectomy. She was admitted to intensive care unit after a long procedure where she received noradrenaline support for 48 hours and transfused two units of blood before discharging her back to the ward. She made an uneventful recovery before being discharged with a long-term catheter and an outpatient appointment for cystogram and chemotherapy. Patient was followed-up on July 2012 after satisfactory cystogram and again on October 2012 by consultant surgeon. She was doing well and managed to maintain her weight. The final histology confirmed a moderately differentiated adenocarcinoma of colonic origin (pT4 pNo) invading the detrusor muscle of the bladder. The myometrium, both adnexa and cervix were unremarkable.

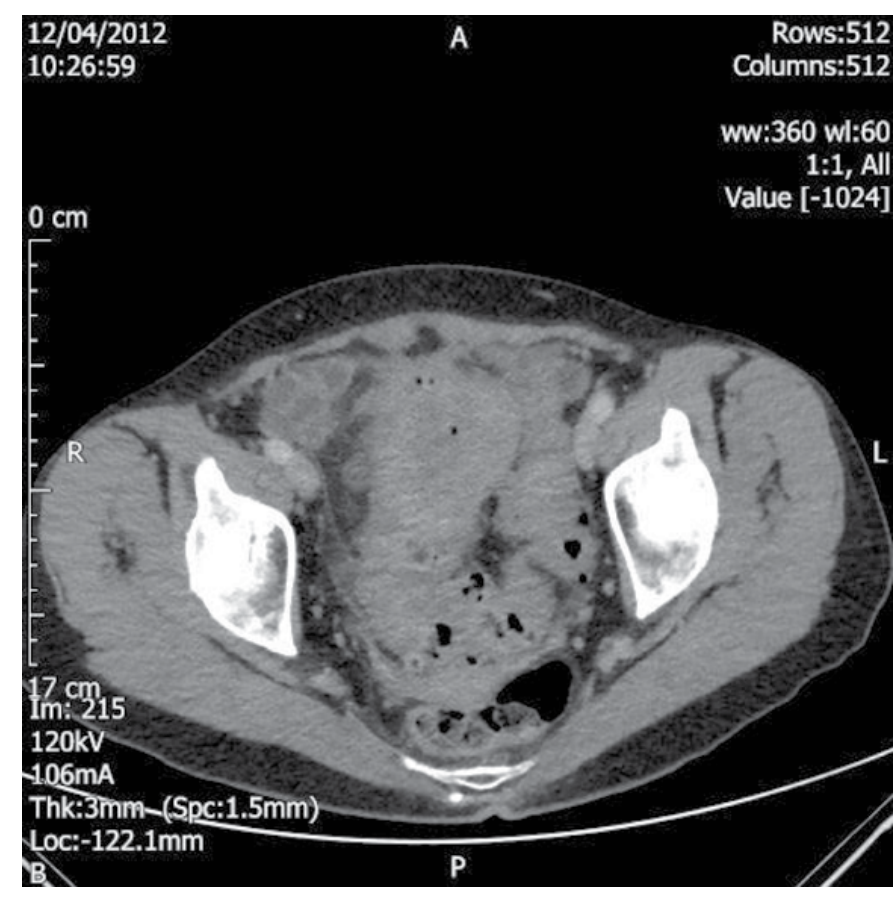

Figure 1: Thickened wall of sigmoid colon and area of possible perforation with gas bubbles.

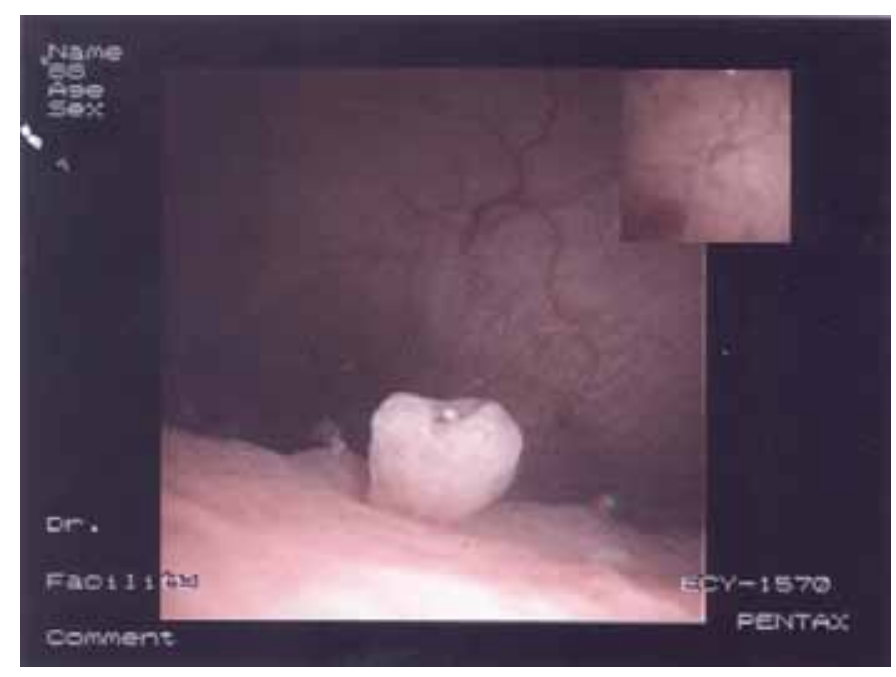

Figure 2: Cystoscopic picture demonstrating a lesion at the trigone. 


\section{DISCUSSION}

Adenocarcinoma of the urinary bladder is uncommon, representing less than $2 \%$ of malignant neoplasms at this site. Adenocarcinoma can arise anywhere in the urinary bladder. Nevertheless, in most cases they involve the trigone and posterior bladder wall [1]. About two-thirds of these tumors present as solitary, discrete lesions, unlike the 'usual' urothelial carcinomas, which tend to be multifocal [2]. These tumors are more common in men with a male-female ratio of $3: 1$. It is usually occurring in the fifth to seventh decades of life. Direct extension of adenocarcinoma from adjacent organs such as the prostate, colon or ovary is more common than primary adenocarcinoma of the urethra or urinary bladder [3]. Metastatic lesions to urinary bladder often infiltrate the wall of the bladder rather than ulcerating the mucosa. In some cases, the bladder is the only genitourinary organ involved in metastasis, mainly from the breast. Most of these patients with urinary bladder metastases are asymptomatic. Symptoms produced by these metastases occur only when the bladder mucosa is involved [4]. These tumors show varied histological pictures and degrees of differentiation. Primary adenocarcinoma of the urinary bladder exhibit several histological subtypes: glandular not otherwise specified, mucinous (colloid), colonic (enteric) type, signet ring cell, clear cell (mesonephric) type and mixed type [5, 6].The majority of the primary adenocarcinoma is of the enteric type and it is morphologically indistinguishable from metastatic adenocarcinoma of colonic origin when evaluated by the cytological, histopathological, histochemical and immunological techniques. However certain features, including the location of the tumor, growth pattern, and clinical history, have been suggested as useful aides for differentiating metastatic colonic adenocarcinoma from primary enteric-type adenocarcinoma of urinary bladder $[7,8]$. It is important to discriminate primary adenocarcinoma of the urinary tract from metastatic colonic adenocarcinoma because of their differing treatment protocols as well as its morphological similarity. Clinical and radiological correlation and ultimately histological confirmation are strongly recommended for confirmation of the diagnosis especially in cases of unknown primary [9]. Treatment options include: surgery, radiation therapy, immunotherapy and chemotherapy. For small bladder tumors, partial/ segmental cystectomy could be performed. However, for large size tumors radical cystectomy is recommended [10].

\section{CONCLUSION}

The diagnosis of urinary bladder adenocarcinoma raises the challenging question of whether the lesion is primary vesical, urachal in origin or metastatic from a distant or an adjacent organ.

\section{Author Contributions}

Bassem Amr - Substantial contributions to conception and design, Acquisition of data, Analysis and interpretation of data, Drafting the article, Revising it critically for important intellectual content, Final approval of the version to be published

Komal Munir - Aquisition of data, Revising it critically for important intellectual content, Final approval of the version to be published

Natasha Santana-Vaz - Aquisition of data, Revising it critically for important intellectual content, Final approval of the version to be published

Venkateswarlu Velineni - Aquisition of data, Revising it critically for important intellectual content, Final approval of the version to be published

\section{Guarantor}

The corresponding author is the guarantor of submission.

\section{Conflict of Interest}

Authors declare no conflict of interest.

\section{Copyright}

(C) Bassem Amr et al. 2013; This article is distributed under the terms of Creative Commons attribution 3.0 License which permits unrestricted use, distribution and reproduction in any means provided the original authors and original publisher are properly credited. (Please see www.ijcasereportsandimages.com/copyright-policy.php for more information.)

\section{REFERENCES}

1. Gill HS, Dhillon HK, Woodhouse CR. Adenocarcinoma of the urinary bladder. Br J Urol 1989;64(2):138-42.

2. Thomas DG, Ward AM, Williams JL. A study of 52 cases of adenocarcinoma of the bladder. Br J Urol 1971;43(1):4-15.

3. El-Bolkainy MN, Mokhtar NM, Ghoneim MA, Hussein MH. The impact of schistomiasis on the pathology of bladder carcinoma. Cancer 1981;48(12):2643-8.

4. ALLEN TD, HENDERSON BW. Adenocarcinoma of the bladder. J Urol 1965;93:50-6.

5. Wang HL, Lu DW, Yerian LM, et al. Immunohistochemical distinction between primary adenocarcinoma of the bladder and secondary colorectal adenocarcinoma. Am J Surg Pathol 2001;25(11):1380-7.

6. Torenbeek R, Lagendijk JH, Van Diest PJ, Bril H, van de Molengraft FJ, Meijer CJ. Value of a panel of antibodies to identify the primary origin of adenocarcinomas presenting as bladder carcinoma. Histopathology 1998;32(1):20-7.

7. Bennett JK, Wheatley JK, Walton KN. 10-year experience with adenocarcinoma of the bladder. J Urol 1984;131(2):262-3. 
8. Melicow MM. Tumors of the urinary bladder: a clinicopathological analysis of over 2500 specimens and biopsies. J Urol 1955;74(4):498-521.

9. Perez-Mesa C, Pickren JW, Woodruff MN, Mohallatee A. Metastatic carcinoma of the urinary bladder from primary tumors in the mammary gland of female patients. Surg Gynecol Obstet 1965;121(4):813-8.
10. Grignon DJ, Ro JY, Ayala AG, Johnson DE, Ordóñez NG. Primary adenocarcinoma of the urinary bladder: a clinicopathologic analysis of 72 cases. Cancer 1991;67(8):2165-72.
Access full text article on other devices

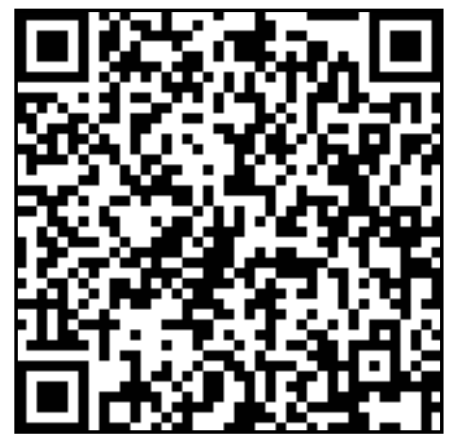

Access PDF of article on other devices

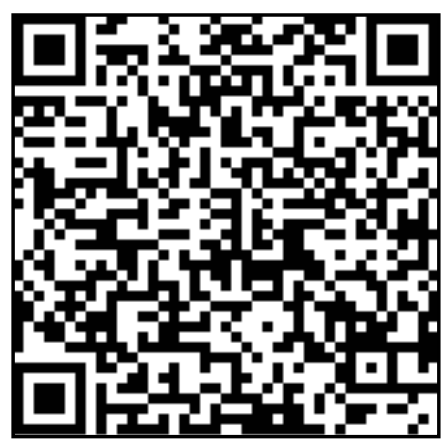

